\title{
A Standardization Strategy that Matches Enterprise Operation
}

James G. Nell

U.S. DEPARTMENT OF COMMERCE

Technology Administration

National institute of Standards

and Technology

Bldg. 220, Rm. A127

Gaithersburg, MD 20899-0001

QC 



\section{A Standardization Strategy that Matches Enterprise Operation}

James G. Nell

U.S. DEPARTMENT OF COMMERCE Technology Administration National Institute of Standards and Technology

Bidg. 220, Rm. A127

Gaithersburg, MD 20899-0001

August 1997

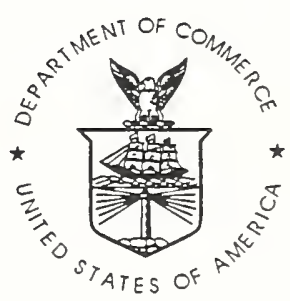

U.S. DEPARTMENT OF COMMERCE William M. Daley, Secretary

TECHNOLOGY ADMINISTRATION

Gary Bachula, Acting Under Secretary for Technology

NATIONAL INSTITUTE OF STANDARDS

AND TECHNOLOGY

Robert E. Hebner, Acting Director 



\title{
A Standardization Strategy that Matches Enterprise Operation
}

\author{
James G. Nell \\ National Institute of Standards and Technology; Bldg. 220, Rm. A127 \\ Gaithersburg MD, USA 20899 \\ +13019755748(V), +13012589749(F),nell@nist.gov
}

\begin{abstract}
This paper presents an analysis of what it means for an enterprise to be integrated, what enterprises are really trying to achieve by integrating their processes, what is really going on among their processes, and how integration may, or may not, change that. Some approaches to analyzing enterprise operation are discussed.

With an idea about how an enterprise really operates, the paper proposes a standards-making philosophy that will improve enterprise operations. The purpose is to create a standardization strategy for integration that matches the operating enterprise. If that is not done, the effort to produce the standards largely will be wasted. This is because an ill-conceived standards strategy will not alter the way enterprises actually operate at the activity level. Finally, there is a suggestion that, perhaps, enterprise-integration-related standards are in a category different from the usual standards for hardware, software, and protocols. Because these standards affect the entire enterprise, and even interacting enterprises, they are more in the category of horizontal standards such as quality and environmental standards.
\end{abstract}

Key words: Enterprise integration, information technology, standards, strategic-standardization management, horizontal standards.

\section{Integrating Enterprises}

Enterprise integration is an improvement concept, as are total quality and error-free performance. These concepts have proven to be very difficult to implement successfully because the desired degree of improvement is difficult to achieve. One part of the difficulty lies in the sheer complexity of changing almost every sector of the business to achieve whatever goal is set. Another part of the difficulty is in assigning metrics to measure progress. Still another part is due to the fact that these concepts do not refer to singularities. Any evaluation of progress is only a glimpse or a snapshot in a continuous stream of miniimprovements that happens over a period of many years. Management has been trying for better quality, higher reliability, and better communication ever since there has been business for profit. There is always room for improvement when considering quality, reliability, and communication among engineering, manufacturing, suppliers, and customers.

Enterprise integration has similar characteristics. Success is conditional in that it involves technological improvement, revising some principles, and the presence of a compatible infrastructure. Enterprise integration also requires a compatible infrastructure in other organizations, so that the objective of the investment is fully utilized. However, two businesses probably would not invest in synchronism, for many reasons. If compatible infrastructure does not exist, the investments in the improvements in one enterprise are difficult to justify. Also, management finds that it is very difficult to translate these investments into some incremental improvement in specific business-improvement strategies, such as lower product cost, lower cycle time, and higher quality. 
On the other hand, some businesses have invested to overcome integration barriers, and have achieved their integration, cost, and quality goals and there was enormous improvement. The products were produced correctly, the first time, on time, but at such a low cost that the facilities and people that were required in the old system were no longer cost effective and justifiable. Their reaction to having underutilized resources was not positive. The solution was to undo the improvement, lose a facility, and terminate people, rather than change old attitudes and management metrics! In these situations, applying technology and meeting cost and quality goals are necessary but not sufficient for success because the enterprise could not overcome the old corporate culture. These cultural attitudes must be kept in mind when analyzing how enterprises really operate.

Even with the barriers, there exists in industry an eagerness to achieve the benefits implied by the words enterprise integration, but with a hesitancy to invest in it. A lot of money has been invested in the past with little perceived results. But, since enterprise integration is a continuum, rather than a specific, singular goal, and since the task is so large and so enterprise wide, the incremental improvements that have been made are largely unappreciated.

In a typical scenario, enterprises evaluate their needs, establish their character, and set goals to achieve market aspirations at an executive-level function often called strategic planning. The enterprise is a set of processes and infrastructure consisting of systems that employ certain technologies. If new needs and goals require new or upgraded systems or processes, and if there is a limited amount of financial means to acquire new capability, then a trade-off analysis will assign priorities, and investments will be made accordingly. There are hundreds of choices that could be made. If we multiply this one scenario by all of the enterprises that are making the same kind of decision there is a very low probability that any two enterprises will have invested the same amounts of money on the same improvements at the same time. Their infrastructure improvements and process improvements will evolve at different rates. Therefore, what results are many enterprises with sets of processes and infrastructure that are not interchangeable, not interoperable, or at the same level of process granularity.

This is also true of organizational functions within a single enterprise [NELL96]. Businesses typically consist of groups, divisions, departments, and sections. Somewhere in that hierarchy the organization becomes function oriented and the executive establishes incentives to subordinate managers to reduce the costs of their function. When that happens there is no advantage to doing things for the benefit of the enterprise because the resulting process and infrastructure improvements tend to suboptimize and even conflict with similar investments in other departments. This is a cultural problem that will exist whether or not there are standards recommending otherwise. Enterprises are therefore not able to migrate toward one another. The fact that enterprises or processes do not share information well is not surprising.

Enterprises say that they wish to be integrated. What does this mean really? It may mean to an enterprise that has tried to integrate, that management is disenchanted with the integration process and has a greater reluctance to invest in integration technologies. Often, managers fail to see in the above scenario that integration is neither a one-time activity, nor a singular achievement, nor a final state. There are hundreds of areas to change that will achieve an improved degree of integration, and there is not enough money to do it all. Even if the money were there and the investment made, there would be a better solution shortly after the investment because technology will not stop advancing. As commerce becomes more global, and there is need to interact with offshore partners as well as domestic partners, the problem compounds because decisions made within one enterprise often do not coincide with the decisions made in other enterprises.

To produce an output, an enterprise or a process usually needs to work with another enterprise or process. To work with can mean several things. Materials and tools need to get there. Parts and components need to get there. Physical things need to exit. Timing is important. In addition, triggering, instructions, controls, intended and actual output, verification, and many other things require that a significant amount of information flows into, and out of, the process. Here is where the going becomes difficult. Judgement enters the process and the possibility of misunderstanding, or miscommunicating, is possible. In fact, miscommunication is probable and to establish a smoothly running system of processes, methods to resolve the miscommunications must be planned into enterprise operation. 
The plan to resolve the miscommunications can take several tracks, but two or three seem to predominate. The first has to do with changing the world by prescribing that all of the hardware and software shall be from the same manufacturer to prevent miscommunications. This approach rarely works because the communications come from people in the enterprise, not from the hardware and software. A related approach is to force all of the possible interactions into a predetermined set of instructions, queries, and responses. It is very difficult to, a priori, prescribe all the possible instructions, queries, and responses. As a result the software engineers are continuously busy adding code to the process software until the system is too ponderous to be reasonably productive. Often in this model the solutions are legislated, in effect, by developing top-down, enterprise-specific standards that must be uniformly adapted by all processes, planned and existing, that contribute to enterprise output. Everyone must be on the same version of the same standard, or provide some kind of conversion or translators; that is, insuring forward and backward compatibility. This may work better for some areas of the information-technology domain than in other areas.

Another way to approach this problem of communication is to analyze how communications have been handled in the past, before computers, and realize that humans negotiated understanding through repeated questions and clarifications. One of the problems in the enterprise-integration domain is that humans and computers must interact and that their brains think differently. So far, the solution has been to make humans interact with computer systems on the computer's terms rather that making computers interact on human terms. People really need smarter computers not faster ones. On a computer's terms all communication must be in precise language and entered through the human machine interface without a single error. Well, humans just do not work that way. Humans tend to create, tinker, modify, and innovate-an iterative process toward some goal.

So, using the humans-only model, what must happen to assure proper information flow? Proper information has been flowing between processes for hundreds of years--or else manufacturing enterprises would have produced no products. Factory communications among humans will involve a minimum of words and a lot of common-sense information that is taken for granted in human discourse. When an engineer hands a drawing of a part to someone in operations to have the part built, the drawing contains symbols referring to the part, but the amount of information on the drawing is very small compared to the amount of information that is transferred (implied and inferred). Most of the information transferred is implicit in the everyday knowledge that humans bring to bear. This is in addition to the expertise that is shared among humans. The expertise, acquired from formal education and experience, enables the human element within the two processes to imply and infer what they need from the information that is contained in the symbols on the drawing, as well as from the information that is not there.

However, in the human-driven processes, there is always an opportunity for additional transfer of information that completes the information exchange. This is the information in the oral question-andanswer negotiation that precedes complete understanding during the inter-process transfer. When a receiving process, such as an operations person, completely understands the concept intended by a sending person, such as a design engineer, a kind of process integration has occurred. This integration will recur, one process at a time, through the product-life history. Enterprise integrators are trying to use informationtechnology tools to improve interprocess interactions; that is, make the information interchange repeatable, faster, more accurate, and computer sensible. Perhaps there is a software model that will achieve necessary integration, improve on the human interactions, but not require a top-down or up-front, complete, and errorfree definition in computer terms.

It is possible to achieve lights-out factories and totally automatic processes. However these phenomena are usually an extension of a human-driven enterprise someplace and are a very small part of the life history of a product. Until major parts of a product-life history can be automated, enterprises and a majority of the processes will act largely as if humans were communicating. If this were the case it would not be productive to assume the enterprise runs as if it were totally automated. Negotiation of some aspect of inter-process communications will be the rule rather than the exception. With the enterprise viewed this way it may be more useful to create a strategy for a more automated enterprise to accommodate this reality, rather than to assume that another standards model is better because it reflects the way analysts wish the 
enterprise were. The necessary interprocess transactions will require the hardware to be physically compatible, the software to interact predictably, the communications protocols to be usable jointly, and the information to be mutually understandable. Achieving this will require a delicate balance of human planning, technology, and a properly designed standards strategy. There also will have to be a mechanism to allow an enterprise to have an internally designed architecture that allows the processes to negotiate the best way to interoperate, among themselves, in a standard way, using standard infrastructure elements.

\section{Standardization}

Having determined how enterprises will operate and produce an output, one can analyze how to design a system of realistic standards to boost process interoperability without stifling technology development and technology insertion. This standards view will involve a standards system that will allow enterprises to operate, change, and grow; and it will improve operations rather than hinder them.

To determine the standards that are necessary depends on existing technology and the standards that are in use [NELL96]. The analysis will be aided by first developing a set of enterprise models. The models should show:

- How much of the information at the interfaces between applications is in an open format

- How much of the semantics is in the one-name-one-meaning category

- How compatible is the hardware

- How well defined are the software interrelationships

- Are the communication protocols agreed upon

- Is there a mutual understanding among the information architectures that are attempting to communicate

Even with all of that defined, the business rules must be compatible, government regulations for commerce must be agreeable, and the people of the enterprise must truly want integration. This does not mean to suggest that the idea of a standard enterprise is realistic. Businesses must be free to design the enterprise of their choice, and with this freedom acceptable integration still must be achievable.

The issue of what things should be standardized and what should be good software design is difficult because the resolution lies in a range between having a few low-level standards and constraining everything with standards. Often the easiest and least-effective solution to a computer-compatibility problem is to standardize everything; the models, the modeling, the tools, and the processes. The loss of user delight, the loss of opportunity to use tools especially designed for the job, and the lost opportunity for technological improvement are enormous. It would stay that way because standards always seem to consume more time to develop than does the technology that standards are attempting to constrain. Shortening the time it takes to make standards is not necessarily a good idea. Imagine a world with many easily and hastily constructed standards that are ill-conceived in their development.

Properly modeling the enterprise will allow more consistent modularization so that enterprises can effectively plug-and-play their processes. The models will ameliorate the need to develop the entire system at one time. Simulation will be possible allowing one to evaluate interoperation among interenterprise entities and to evaluate systems with differing granularity. Enterprises will be able to plan migration paths more effectively. Information should be a separate asset, so that changing applications will be possible without re-entering information about the products and processes unnecessarily. Enterprises can define paths using standards and enterprise models to make the product and process information tie logically into enterprise goals, strategies, capabilities, and business rules. The models and standards should be scalable so that a high-level model is essentially the same as a lower-level model; that is, they should use the same or compatible modeling constructs for all levels.

Rather than setting specific limits to everything, such a standards set should be designed to force enterprises to analyze and document, perhaps electronically on-line, what they do; and then operate their enterprise accordingly. Lower-level standards would still define interfaces and the supplying process would 
indicate which standards apply for that particular communication. They would also share, on-line, the set of information they need to know about their processes. This way, an enterprise shopping for a process would be able to evaluate, on-line, that a process meets both quality and integration guidelines and that they conform to listed interface standards. The process in the shopping enterprise could then make any needed adjustments to itself and to the appropriate enterprise model (function, hardware, software, communication, and information), establish a relationship, and operate in an acceptable (to both parties) level of integration.

Are there special standards required to enable virtual enterprises? A virtual enterprise, or an extended enterprise, is an enterprise--not really different from a traditional enterprise other than the fact that it can append and shed processes quickly. There are more legal and regulatory issues than technical issues when removing barriers to virtual-enterprise operations. Technically the standards categories involved are the same: hardware, software, communications, information, and architecture. The notion of virtual enterprise brings in a time element such that the enterprise can form or dissolve very quickly. To accomplish this, standards other than just technical ones must be in place. A virtual-enterprise candidate may have to negotiate such contracts as a basic-ordering agreement in advance of considering such an alliance. Collaborating enterprises must resolve other things in a generic sense long before true virtual-enterprise commerce can succeed. Eventual parties comprising the enterprise will probably pre-define the following, currently often locally unique: financial standards, environmental-impact standards, markup language, safety requirements, statutes, product liability, quality, and organizational requirements.

Standardizing the enterprises, parts of the enterprise, the products, the information transferred, and the processes, is probably not going to be a productive use of standard-making resources. What seems more useful is to standardize the interfaces, the nomenclature, and the formats and allow the enterprise-tool builders to use these standards to design software in such a way as to allow the processes to communicate.

\section{Strategic Standardization}

What is the best way to employ standards in an enterprise? Many executives feel that funding standards development is a non-value-added activity, and that paying extra money to have standards incorporated in the tools they buy is an increase in process cost. They feel that both are a cost of doing business and this cost should be minimized [HOW96]. This could be a valid course of action for enterprises that plan to maintain control of all processes that produce their product, sell their product locally, and face limited competition. Enterprises that plan to produce and market their product globally may take a different view.

For the global enterprise a better approach to planning for standards may be to include standardization as a component of their strategy [BET95]. These enterprises would be planning to operate on a more global basis, planning to compete more effectively in a new market, seeking to expand their market or their competitive position, and seeking ways to protect significant investment in rapidly changing technology. These investments could be in their manufacturing facilities, quality assurance and environmental impact policies and procedures, and process-interoperability plans. The goal of strategic standardization is to see how certain standards would help the enterprise better fulfil its mission. One or more of these strategies could be invalid or prohibitively difficult and expensive if, at the same time, the company did not investigate ways to mitigate barriers that could impede success because sufficient attention was not paid to standards. Analyzing these strategies properly can indicate marketing opportunities or probable cost saving. Cost saving could come from leverage achieved through working with others to achieve a shared advantage in some area of commerce; either to improve infrastructure, process interoperability, or the operation of virtual (or extended) enterprises. The marketing opportunities would arise from government or trading-bloc requirements to require quality, environmental, and/or recycling standards as a hurdle-of-entry into that market.

In applying strategic-standardization management, the standards-investment decision becomes a strategic one for the enterprise rather than a tactical one for the process. Planners must evaluate what they are trying to accomplish, which standards would make the job easier, and the best way to get those standards implemented. This would involve standards development as well as lobbying to gain acceptance. Strategicstandardization management is not about standards. Rather, it is about leveraging all aspects of the standardizing process to optimize competitiveness. This should be a horizontal discipline, that is, enterprise 

wide rather than applicable to a single product or process. Example horizontal strategies include quality, environmental, electronic-data interchange, and safety. Parallel with this horizontal-enterprise strategy, a new category of standard has emerged--often referred to as horizontal standards. Rather than affecting one process or tool these standards affect everything that an enterprise does within the subject area of the standard. All of the enterprise processes and the infrastructure that supports them would be affected. Therefore to position the enterprise to best deal with the standard requires executive-level decision making during the strategy-making process and not disjointed point solutions. The philosophy of some of these horizontal standards is slightly different from the usual standards.

These standards tend to ask what one does rather than to tell everyone what to do. ISO 9000 series requires that for compliance, one must reveal all enterprise-quality-related activities and procedures, document them, implement them as documented, then certify that it is done. Perhaps improving enterprise-process interoperability will require a similar philosophy. Rather than forcing every process that wishes to communicate to have the same everything, maybe the processes involved in the transaction, the querying process and a responding process, could operate by asking rather than telling. To do this, the process initiating the discourse could ask another process, in a standard way, what that process speaks, what its capability is, and whatever else is required. The answering process would respond in a standard way. These interactions could be on a resource such as the Internet. The processes then could determine between themselves the best way to communicate.

Standards at the correct level and constraining the correct things act to preserve innovation incentive. They allow users to form alliances more easily and with fewer barriers, and they allow more effective product and process differentiation; that is, they enable commerce to be effective and competitive. With more deregulation, companies are viewing standardization more globally, and at a strategic perspective rather than at a tactical perspective. Without the strategic viewpoint, standards can be a barrier to global operations because different markets have legally enforceable entry requirements that must be addressed at the corporate or government level.

\section{Conclusions}

Cultural inertia, direction and rate of enterprise change, and human ingenuity will limit the effectiveness and use of standards to constrain enterprise design. Defining and representing the interfaces that exist in a manner similar to the ISO 9000 series of standards may enable enterprises to communicate without standardizing an enterprise or process structure. The process of evaluating how best to communicate electronically will involve some tradeoffs. Enterprise designers must evaluate whether the technology used to achieve acceptable functionality has a life that is shorter than the time it takes to develop and/or modify a standard to constrain the technology. The alternative to standards is to use the technology to circumvent the problem.

There are several areas to evaluate when analyzing enterprise operation. If sets of models from one enterprise are to connect to models of another enterprise, both sets of models must have considered the same areas or the models probably will not interoperate. To ascertain whether modelers can manage these areas consistently, informally from a checklist or formally by standards, needs further implementation experience and discussion to decide. The issue needs satisfactory resolution because the areas in question are precisely the interfaces that one enterprise will mediate with another to communicate.

Areas of integration technology needing standards-like attention are hardware, software, communications protocols, information, and architectures. Enterprise-representation technology, coordinated terminology, and enterprise-representation architectures are not yet adequately addressed. There are a few areas that appear to be good candidates for standards. One is terminology and another is enterprise models. The solution to the terminology problem appears to lie in some registry to which an individual enterprise can map its terminology. Properly constraining enterprise models and the way they communicate among themselves, would reveal, in a standard way, the necessary points from which one enterprise architecture can mediate an information transfer with another enterprise architecture. 

Ensuing enterprise representation standards should not mandate standard processes, standard enterprises, standard organizations, standard methodologies, or standard-enterprise data. Enterprise-representation standards should define the key ideas involved with enterprise frameworks or enterprise models so that the models produced are consistent. Then, vendors can develop tools that produce and operate frameworks and models with minimum investment risk and with the maximum confidence that they will encounter known interfaces. Ideally, standards should enable interoperability and still protect innovation, efficiency of approach, and migration capability.

Interprocess communication may not be $100 \%$. Well, it rarely ever was, is not now, and maybe never will be the case that everyone will be invested to the same level and version to enable $100 \%$ communication on the first try. Let us recognize that fact and find a way to communicate to the best of our ability. It may require a change in standardization philosophy to move toward ask rather than tell standards.

Governments around the world use standards in a strategic sense; for example, as a barrier to product entry. A corresponding strategic view will help companies to operate better in global environments. Businesses must pay closer attention to standards matters to insure that they will be able to compete on a playing field that is more level. Many standards issues are becoming more company or industry wide, such as quality, environmental, services, and electronic-data interchange. Continued participation in standards activities and strategic treatment are needed to avoid standards that add cost but do not add value, and to avoid standards that are being developed deliberately for the purpose of restraining trade.

\section{References}

[BET95] Betancourt, Diego, and Walsh, Robert, "The Evolution of Strategic Standardization Management", StandardView, Vol. 3, No. 3, 1995-September.

[HOW96] Howie, Robert L., Jr., "Competition 2000", Special Advertising Section, Business Week, 1996October-21.

[NELL96] Nell, James G., "Enterprise Representation: An Analysis of Standards Issues", Modeling and Methodologies for Enterprise Integration, Edited by Bernus and Nemes, Chapman and Hall, 1996

The author acknowledges contributions to this paper from Shirley Hurwitz, Neil Christopher, Howard Bloom, and Kurt Kosanke. NIST, through its SIMA (Systems Integration for Manufacturing Program) sponsored this paper and the NIST participation in the ICEIMT'97 activities. Since the U.S. part of the ICEIMT'97 activities is funded by the United States government, this paper is not subject to copyright. 


